\title{
INCREASED BUCCOLINGUAL DIMENSION OF MAXILLARY SECOND PREMOLAR BRACKET : IS IT NECESSARY?
}

\author{
Fady Hussein Fahim* and Dina Osman ElAbbasy*
}

\begin{abstract}
Introduction : Small-sized maxillary second premolars pose a challenge for the orthodontist with regards to alignment of the contact point with the maxillary first molar. To overcome this discrepancy, Dentaurum Discovery Smart brackets have been developed with thicker buccopalatal dimension.
\end{abstract}

Aim of the study : The aim of this study was to compare between two bracket systems (Dentaurum Discovery Smart) and conventional (American Orthodontics/Ormco) brackets regarding the effect of differential in-out buccopalatal thickness on the alignment of the contact points of maxillary premolars and molars as well as bond failure rate.

Materials and Methods : This prospective study consisted of 24 patients with an age range of 20-30 years old and a mean age of 24.5 years selected from a private practice in Cairo, Egypt.

The patients had full permanent dentition and were non-syndromic with no previous orthodontic treatment. At least one maxillary second premolar had a reduced buccopalatal dimension. The patients were randomly divided into two groups, each group containing 12 patients. Group 1 were treated with Discovery smart Dentaurum brackets and Group 2 were treated with American Orthodontics /Ormco mini diamond brackets. Treatment was continued with fixed appliances for 2 years until rectangular stainless steel wires were reached.

Results : There was a statistically significant difference between Group 1 and Group 2 regarding proximal contact point discrepancy $(\mathrm{P}<0.05)$ with $50 \%$ of Group 2 having at least $0.5 \mathrm{~mm}$ buccal contact point discrepancy between upper second premolar and upper permanent first molar. No contact point discrepancy was observed for Group $1(0 \%)$. However bond failure rate was significantly higher in Group 1 (80\%) compared to (20\%) in Group 2.

Conclusions : The Dentaurum Discovery Smart brackets are highly effective in achieving adequate buccopalatal alignment of small sized maxillary second premolars with maxillary first premolars and permanent first molars. However they have the drawback of frequent bond failures due to their thicker buccopalatal dimension.

\footnotetext{
* Lecturers of Orthodontics, Faculty of Dentistry, Cairo University
} 


\section{INTRODUCTION}

Orthodontic finishing and detailing is regarded as a challenge for the orthodontist (Karad, 2006). One of the main advantages of the preadjusted edgewise appliance is the provision of improved quality of orthodontic treatment results and finishing. However, the great diversity of the patients' dental characteristics and malocclusion limits the orthodontist's ability to achieve optimum results. Moreover, the individual variations related to precise bonding of the orthodontic brackets can also affect the accuracy and excellence of finishing (Koo et al, 1999 and Poling, 1999).

Achieving optimal results for each patient should be included in every orthodontist's treatment plan by beginning with the end in mind. This helps the orthodontist formulate a customized selection of orthodontic appliances and suitable mechanics to fit individual variations within each patient. Following a finishing checklist is important as a guide to help the orthodontist achieve an optimum result. The checklist constitutes significant elements which are oral health, esthetics, occlusion, function, normal peridontium and stability (Roth, 1981; McLaughlin \& Bennett 2003 and Mucha, 2018).

Additionally the ABO Model Grading system for scoring dental casts and orthopantograms is a valid one and was introduced to help orthodontists achieve a more objective clinical examination. It consists of eight criteria as follows: alignment, marginal ridges, buccolingual inclination, occlusal relationships, occlusal contacts, overjet, interproximal contacts, and root angulation (Gottlieb, 1975 and Casko et al, 1998)

Alignment is a principal purpose of any orthodontic treatment plan. In the maxillary arch, the central grooves of the premolars and molars are utilized to evaluate the quality of the alignment in the posterior region (Eismann, 1974).

Achieving ideal Class I occlusal relationship based on Angle's classification entails that the buc- cal cusps of maxillary molars, premolars and canines must align within $1 \mathrm{~mm}$ of the interproximal embrasures of the mandibular posterior teeth. Furthermore, the mesiobuccal cusp of the maxillary first molar must align within $1 \mathrm{~mm}$ of the buccal groove of the mandibular first molar (Angle, 1907). Following Andrews six keys of normal occlusion is important as well for attaining optimum finishing results (Andrews, 1972). Ricketts, 1969 indicated the importance of the contact position of the maxillary second bicuspid and considered it pivotal to a successfully treated malocclusion. Ideally the central grooves (mesio-distal) of maxillary posterior teeth should be in the same plane or alignment. It is not uncommon to observe that the maxillary second premolar bucco-palatal dimension is smaller than the maxillary first premolar teeth (Bishara and Jakobsen, 1989). This frequently happens due to genetic and environmental factors (Bailit, 1975) Thus it was postulated that if both brackets had the same bucco-palatal thickness, the maxillary second premolar will finish buccal to maxillary first molars ((Karad, 2006 and Mucha, 2018).

Dentaurum produced the Discovery smart brackets with differential in/out or bucco-palatal thickness between the maxillary first and second premolar brackets. It was assumed that this will compensate the difference in bucco-palatal dimension of maxillary first and second premolar teeth.

Therefore, this study was conducted to compare between two bracket systems: First system with differential bracket thickness (Dentaurum Discovery Smart) and other systems with similar bracket thickness (American Orthodontics, Ormco mini diamond) regarding the accuracy of alignment of proximal contact points for maxillary premolars and molars.

\section{SUBJECTS AND METHODS}

In a prospective non-randomized study, twenty four patients that fulfilled the inclusion/ exclusion criteria were selected in the sample. 12 patients 


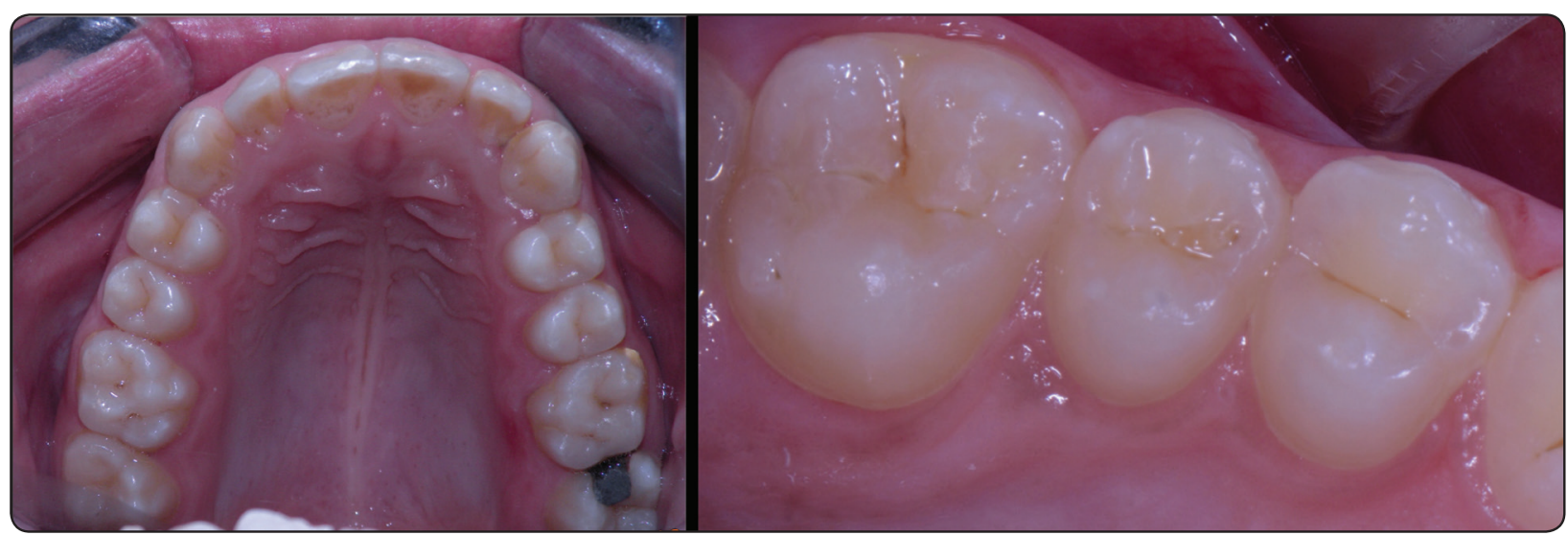

Fig. (1) Showing upper second premolar with a small buccopalatal thickness compared to upper first premolar.

were non-randomly allocated in two groups.

\section{Inclusion criteria:}

1. Full permanent dentition.

2. The Bucco-palatal dimension of maxillary second premolars (U5) smaller than that of maxillary first premolar (U4) (Figure 1)

3. Non-syndromic.

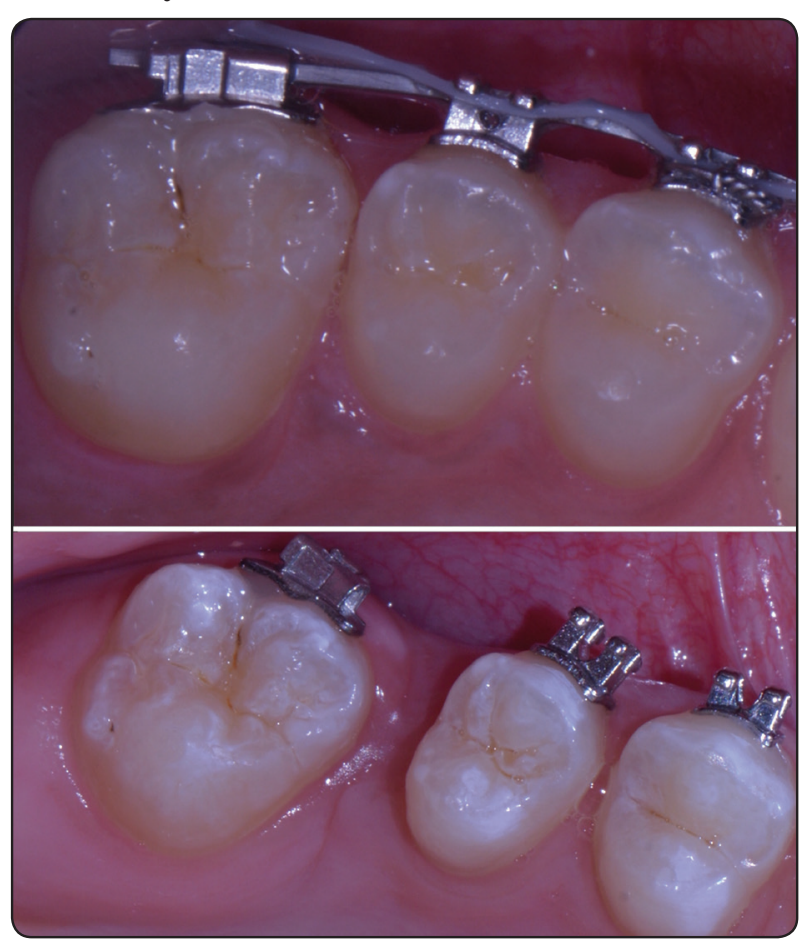

Fig. (2) Showing the thicker buccopalatal dimension of the Discovery bracket on U5 compared to conventional Ormco/American Orthodontics bracket on U4

\section{Exclusion criteria}

1. Mixed dentition.

2. The Bucco-palatal dimension of maxillary second premolars were same as that of maxillary first premolar.

3. Previous orthodontic treatment.

The first group (Gp 1) were treated with Discovery smart Dentaurum brackets (Figure 2) while the second group (Gp 2) were treated with American Orthodontics /Ormco mini diamond brackets (Figure 3).

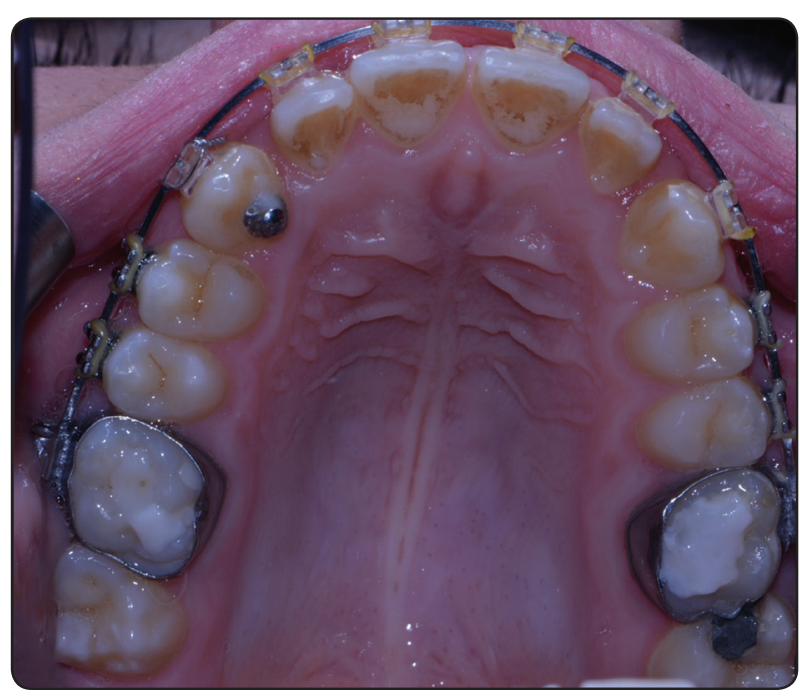

Fig. (3) Showing the conventional U5 brackets of American orthodontics/Ormco with regular buccopalatal thickness as $\mathrm{U} 4$ 
The bracket specifications were as follows:

Dentaurum catalogue : Discovery smart

U4 bracket in/out $=0.7 \mathrm{~mm}$

U5 bracket in/out $=1.2 \mathrm{~mm}$

Both American Orthodontics/ Ormco U4 and U5 brackets bucco-palatal thickness were the same.

Orthodontic treatment was performed for at least 2 years to ensure complete leveling and alignment before gathering the results.

Archwire sequence used started with $0.016 \mathrm{NiTi}$ then $0.016 \mathrm{SS}$ then $20 \mathrm{SS}$ then $0.019 \times 0.025 \mathrm{SS}$. (Mandall et al, 2006).

According to American Board of Orthodontics grading system (Casko et al, 1998), if the mesial or distal alignment at any of the contact points is $0.50 \mathrm{~mm}$ to $1 \mathrm{~mm}$ deviated from proper alignment, 1 point shall be scored for the tooth that is out of alignment .If the discrepancy at the contact point is greater than $1 \mathrm{~mm}$ then 2 points will be scored for that tooth (Figure 4).

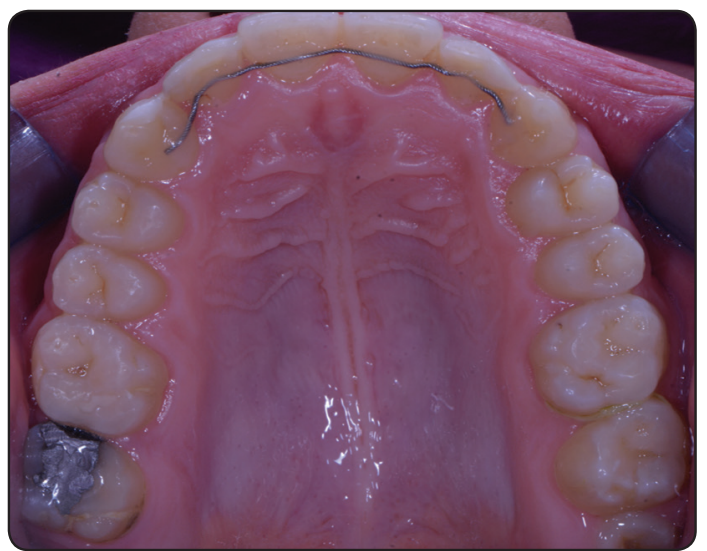

Fig. (4) Showing the buccal contact point displacement of U5 in relation to U6 as a result of using conventional premolar brackets (American Orthodontics or Ormco)

The number of bracket bonding failures were recorded for each group through the 2-years treatment (Figure 5).
No finishing bends (step in/ out bends) were done during the duration of the treatment for either group.

The post-treatment plaster models for each patient was analyzed utilizing the $\mathrm{ABO}$ gauge and scores were given based on contact points discrepancies between the U5 and U6 (maxillary first permanent molar).

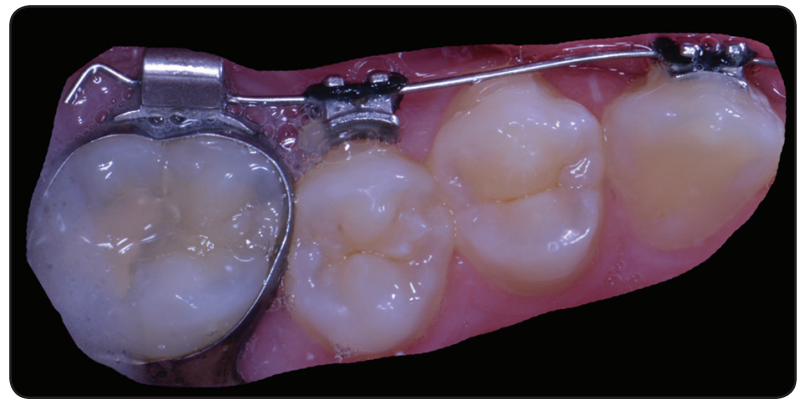

Fig. (5) Showing U5 bracket failure of Dentaurum Discovery Smart bracket

\section{RESULTS}

\section{Statistical method}

All Data were collected, tabulated and subjected to statistical analysis. Statistical analysis was performed by SPSS in general (version 17).

Qualitative categorical variables were described by proportions and percentages.

Fisher exact test was applied for 2 by 2 contingency tables.

Significance level was considered at $\mathrm{P}<0.05(\mathrm{~S})$; while for $\mathrm{P}<0.01$ was considered highly significant (HS). Two Tailed tests were assumed throughout the analysis for all statistical tests.

Statistical results are shown in Tables 1 and 2. 
TABLE (1) Discrepancy in alignment of contact points between the maxillary second premolar and maxillary first permanent molar brackets (American Orthodontic bracket and Discovery Smart bracket)

\begin{tabular}{|c|c|c|c|c|}
\hline & \multicolumn{2}{|c|}{ NO } & \multicolumn{2}{c|}{ Yes } \\
\cline { 2 - 5 } & Frequency & Percent & Frequency & Percent \\
\hline $\begin{array}{c}\text { American } \\
\text { Orthodontics }\end{array}$ & 5 & $\% 50$ & 5 & $\% 50$ \\
\hline $\begin{array}{c}\text { Discovery } \\
\text { smart }\end{array}$ & 10 & $\% 100$ & 0 & $\% 0$ \\
\hline
\end{tabular}

\section{Fisher's exact test:}

The two-tailed $\mathrm{P}$ value equals 0.0325

\section{$\mathbf{P}<0.05$ Significant}

The association between rows (groups) and columns (outcome) is considered to be statistically significant.

Contact point discrepancies are statistically significantly associated with the type of bracket. It is higher for American Orthodontics brackets compared to Discovery Smart (50\%).

TABLE (2) Comparison between the two study groups regarding U5 bracket failure rate within 2 years treatment period

\begin{tabular}{|c|c|c|c|c|}
\hline & \multicolumn{2}{|c|}{ NO } & \multicolumn{2}{c|}{ Yes } \\
\cline { 2 - 5 } & Frequency & Percent & Frequency & Percent \\
\hline $\begin{array}{c}\text { American/ } \\
\text { Ormco brackets }\end{array}$ & 8 & $80 \%$ & 2 & $20 \%$ \\
\hline $\begin{array}{c}\text { Discovery smart } \\
\text { brackets }\end{array}$ & 2 & $20 \%$ & 8 & $80 \%$ \\
\hline
\end{tabular}

\section{Fisher's exact test :}

The two-tailed $\mathrm{P}$ value equals 0.0230

\section{$\mathbf{P}<0.05$ Significant}

The association between rows (groups) and columns (outcomes) is considered to be statistically significant.
That means bracket failures are statistically significantly associated with the type of bracket . It is 50\% lower for American Orthodontics

\section{DISCUSSION}

There are certain clinical situations that pose difficulty in obtaining an ideal occlusal result during the finishing stage. To minimize this problem, orthodontists must clearly define their goals while establishing their treatment plan for every case. To evaluate the quality of treatment, a guideline should be followed to assess the quality of the treatment outcome. In this study, the ABO grading system for plaster models and orthopantograms (Casko et al, 1998 and Casko et al, 2000) has been used to quantify static occlusal goals during finishing. Others such as (Margolis, 1997) proposed following a systematic method of analyzing the face and teeth in forming an orthodontic treatment plan. Poling et al, 1999 also proposed following an orthodontic checklist or detailing form where needs for bends and/or wire adjustments are documented.

Variations in tooth structure and size, such as unusual crown shape and size must be taken into consideration during treatment planning as they need some alterations with regards to bracket in-out, tip/ torque and height criteria.

A properly positioned maxillary second premolar is essential to ensure good interdigitation with the mandibular premolars and first permanent molar. The maxillary second premolar usually has some variations in its size and it is quite frequent to observe a smaller buccolingual dimension for this tooth compared to the maxillary first premolar (Kieser et al, 1986). The small size of upper second premolars buccolingually will also be reflected on its size occlusogingivally. Suarez and Vilar, 2010 studied the effect of steady height bracket placement on marginal ridge leveling using digitized models. They concluded that vertical placement bracket protocols, which do not take into consideration individual buccal crown convexities and lengths, may 
propose a preliminary bracket placement error and lead to marginal ridge discrepancies.

During the finishing stage it is crucial to ensure that the marginal ridges of posterior teeth are placed at the same level. This will guarantee a correct position of the cusps and fossae of these teeth. This is significant for a healthy periodontium since it ensures that the cementoenamel junctions are relatively aligned and therefore flat bone levels are obtained interproximally (Summers, 1971 and Karad, 2006)

One of the main cornerstones for ideal finishing is that the central fossae and grooves of maxillary premolars and molars should be well aligned. Also the buccal surfaces of the premolars and first molars should have parallel contours (Andrews, 1972 and Poling, 1999). Properly positioned proximal contact points between upper premolars and molars prohibits the impaction of food and aids in achieving stable occlusal results. These teeth are chosen because they represent the functioning surfaces of the upper posterior teeth and are quite easy to detect clinically. Contact points examination must be done from the buccal as well as the occlusal aspects. A problem arises when the maxillary second premolar is smaller in size buccopalatally than the maxillary first premolar as shown in our study. This can jeopardize the alignment of this tooth buccally as well as the position of its functional palatal cusp. Furthermore, reduced buccopalatal thickness of the upper second premolar causes the upper first molar to rotate mesially thus exaggerating the Class II tendency and resulting in buccal displacement of the second premolar. The discrepancy would be very obvious between the buccal surfaces of the upper second premolar and upper first molar (Karad, 2006).

The soluion to this problem would traditionally include finishing bends and/or bracket repositioning to achieve finishing excellence. Sometimes bracket repositioning does not guarantee achieving the desired position. It also weakens the composite bond of the bracket with the tooth and necessitates etching the tooth surface again. On the other hand finishing bends have the disadvantage of exerting undesired effects on adjacent teeth (Poling, 1999) Hence, the less the need to employ finishing bends in the archwires, as well as reposition the brackets the better and faster the results. To overcome the need for finishing bends, Dentaurum innovated the Discovery bracket system which has a thicker buccolingual profile in the hopes of overcoming this size discrepancy.

The results of this study showed a statistically significant difference between the two study groups whereby the buccopalatal discrepancy between the maxillary second premolars and permanent first molars in Discovery bracket system was zero compared to an $0.5 \mathrm{~mm}$ buccal discrepancy in the American/Ormco bracket system. Out of the total sample, 50\% of American/Ormco bracket system showed contact point discrepancies of the maxillary second premolar and first molar compared to $0 \%$ of Discovery Smart bracket system. On the other hand, the bracket failure rate for Discovery bracket within the 2 years treatment period was $80 \%$ compared to $20 \%$ for the other bracket system. This high failure rate is a disadvantage and is mainly due to the thicker buccopalatal profile which makes it easier to debond with any occlusal forces.

During the finishing stage, paying attention to details is crucial to achieve the best results. It is always recommended to look at the face, teeth and smile not only the orthodontic appliance in order to achieve good esthetics (Mucha, 2018).

\section{CONCLUSIONS}

1. The difference in alignment of contact points between U5 and U6 were statistically significant between the two groups with better alignment results with the Discovery bracket system than the other conventional systems. Therefore better finishing results are achieved. 
2. The increased thickness of maxillary second Discovery Smart brackets increased the frequency of bonding failure.

\section{REFERENCES}

- Andrews LF. The six keys to normal occlusion. Am J Orthod 1972;62:296-309.

- Angle E.H. Malocclusion of the teeth. 7th Ed. Philadelphia. S.S. White and Co., 1907.

- Bailit H L . Dental variation among populations-an anthropologic view. Dental Clinics of North America 1975;19: 125-139.

- Bishara SE, Jakobsen JR. Compensatory developmental interactions in the size of permanent teeth in three contemporary populations. Angle Orthod.1989 Summer; 59(2): 107-12.

- Casko JS, Vaden JL, Kokich VG, Damone J, James RD, Cangialosi TJ, Riolo ML, Owens SE, Bills ED. Objective grading system for dental casts and panoramic radiographs. Am J orthod Dentofacial Orthop 1998; 114: 589-599.

- Casko J, Vaden J, Kokich V. American Board of Orthodontics objective grading system for dental casts and panoramic radiographs. Am J orthod Dentofacial Orthop 2000; 114: 530-532.

- Eismann, D. A method of evaluating efficiency of orthodontic treatment, Trans Europ Orthod Soc, 1974: 223232

- Gottlieb, E . Grading your orthodontic treatment results,. J Clin Orthod, 1975 9:156-161.

- Karad A. Excellence in finishing: Current concepts, goals and mechanics. J Ind Orthod Soc 2006; 39:126-138.
- Kieser JA, Groeneveld HT, Preston CB. On the nonexistence of compensatory tooth size interaction in a contemporary human population. J Dent Res 1986; 65:1105-1107.

- Koo BC, Chung CH, Vanarsdall RL. Comparison of the accuracy of bracket placement between direct and indirect bonding techniques. Am J Orthod Dentofacial Orthop 1999;116:346-51.

- Mandall NA, Lowe C, Worthington HV, Sandler J, Derwent S, Abdi-Oskouei M, Ward S. Which orthodontic archwire sequence? A randomized clinical trial. Eur J Orthod 2006;28(6): 561-6.

- Margolis MJ. Esthetic considerations in orthodontic treatment of adults. Dent Clin North Am 1997;41:29-48.

- McLaughlin RP, Bennett JC. Finishing with the preadjusted orthodontic appliance. Semin Orthod 2003; 9:165-183.

- Mucha JN. Orthodontic finishing: Ten steps to success. APOS Trends Orthod. 2018;8:184-99.

- $\quad$ Poling R. A method of finishing the occlusion. Am J Orthod Dentofacial Orthop 1999;115:476-87

- $\quad$ Ricketts RM. Occlusion - the medium of dentistry. J. Pros. Dent. 1969; 1:39-60.

- $\quad$ Roth RH. Functional occlusion for the orthodontist JCO 1981; 1:32-50.

- Suarez C, Vilar T. The effect of constant height bracket placement on marginal ridge levelling using digitized models. Eur J Orthod 2010;32:100-5.

- $\quad$ Summers C. The occlusal index: a system for identifying and scoring occlusal disorders. Am J Orthod 1971; 59:552-66. 